\title{
Analysis of Different Coverage and Connectivity Techniques
}

\author{
Bhagwan Sahay Meena
}

\begin{abstract}
Wireless sensor networks (WSN) comprises of a number of micro nodes known as sensor nodes which have the capability of sensing, data processing and communication. WSNs have the capacity to connect with their environment through sensors, process data and communicate the data with their neighbors. The fundamental issues in wireless sensor network having great impact on WSN are coverage and connectivity. The paper describes the important issues of wireless sensor network, classification of coverage problem. Some strategies of coverage are also described in which various topologies efficient coverage ratio is found in one of them. Some mechanisms are discussed here which reduces cost and also extend network lifetime. The paper also describes the connection between coverage and connectivity and $3 D$ coverage control algorithm.

Keywords : Coverage, Connectivity, Sleep Scheduling Mechanism, AR-SC problem
\end{abstract}

\section{INTRODUCTION}

The development of modern wireless sensor network has been promoted by the advancement of sensor technology, mod- ern networking and wireless communications technology. The development of low cost, low power, multifunctional sensor nodes of small size and which communicate in short distances is enabled by recent advances in micro-electromechanical sys- tems (MEMS) technology and digital electronics. Throughout the world WSN are currently in concern. WSN technology can be used in military applications, biomedical, disaster relief, environmental testing, health and other fields. WSN technology is regarded as the most prominent technology.

In [2], describes the effects of multidimensional reduction (MDS and ISO-MAP) on 3D localization of nodes in WSN. In wireless sensor network nodes are located randomly in a sensor field. So, coverage is one of the fundamental problems in WSN. WSNs nodes are dropped randomly in the sensor field. Energy is a very limited resource and thus must be used moderately. So, a crucial matter in the model of systems formed on WSNs is energy conservation. We need to use the sensor nodes in an effective way to enlarge the network duration as energy resource is limited. In paper [3], gives the study of the localization accuracy between BLE becons and Smartphones in idle scenario(no user interaction) and with user interaction.

The two important matters for the service's class in the WSN are coverage and connectivity. Coverage is regarded as a measure of service's class for the sensor networks. The

Revised Manuscript Received on July 22, 2019.

Bhagwan Sahay Meena, Assam University, Silchar energy utilization becomes high and decreases the network duration if the service's class parameter is large in number. Energy consumption can measure the service's class of the network. Thus the two major metrics in determining the network's class are coverage and energy consumption. The paper presents the challenges faced by WSN due to the limited energy resource,

process of deployment, sensor nodes transmission and sensing fields.

The brief view of the rest of the paper is: In section 2, two major issues of WSN are covered. In section 3, the coverage problem is classified. In section 4 , different cover- age techniques are described, in section 5 sleep scheduling mechanism and its parts are discussed. In section 6 AR-SC problem and target coverage is discussed. In section 7 coverage and connectivity's connection and in section $8,3 \mathrm{D}$ coverage control algorithm is described.

\section{MAJOR MATTERS FOR WIRELESS SENSOR NETWORK}

\section{A. Coverage}

In a wireless sensor network, coverage is an important issue. Coverage is defined as the region covered by the sensor nodes. In the targeted sensor field, to check whether all points are inside the sensing field of the located sensor is the goal of the coverage problem.

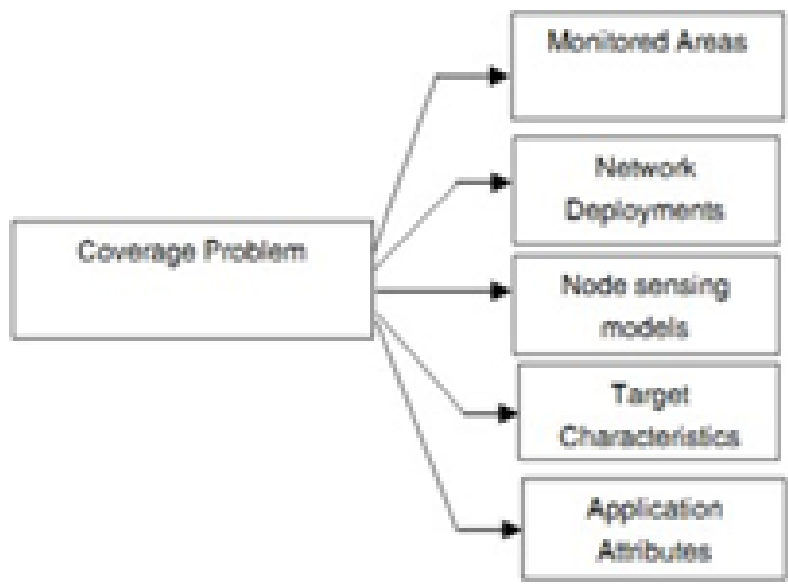

Figure: 1 Categorization of coverage issues

\section{B. Connectivity}

Another major issue of WSN is connectivity. Connectivity requires only the location of one active node among one or more active nodes to be within the communication range such that communication backbone is

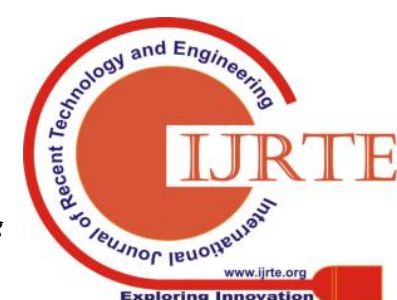


formed by all active nodes. If whole pair of nodes can in any way communicate with other nodes then it is said to be connected. Power consumption is minimum for connectivity. $\mathrm{K}$ connected graph means there is $\mathrm{k}$ paths between every two nodes in which if $\mathrm{k}-1$ nodes is removed, the graph is still connected.

\section{CATEGORIZATION OF COVERAGE PROBLEM}

Here in these section different types of characteristics of coverage is classified.

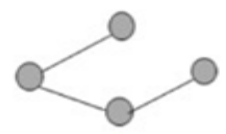

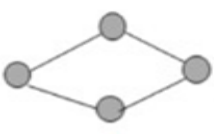

$\mathrm{b}$

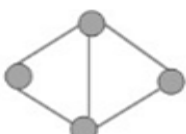

C
Fig. 2. (a) 1-connected (b) 2-connected (c) 3-connected

A. Monitored Areas

i. Area Coverage

ii. Point Coverage

\section{iii. Barrier Coverage}

1) Area Coverage: In area coverage, to cover whole point by at least one sensor in the region is the objective of regional coverage. In regional coverage, the deployment of nodes is dense since each point requires monitoring. Thus the nodes are overlapped and it results in overlapped coverage.

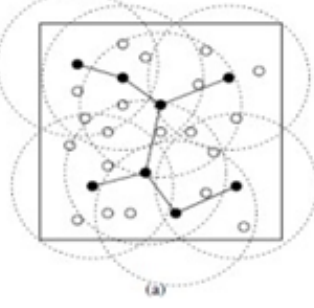

Fig. 3. Area Coverage

2) Point Coverage: In point coverage, minimum number of sensor nodes covers some targets, which can be represented as a given set of discrete points. In point coverage, only some points are covered by the sensor nodes not the total area. Here covering is based on the points which are applicative.

3) Barrier Coverage: Barrier coverage is coverage where a sensor nodes form a barrier for intruders which is the combination of the covered regions of the sensors. Barrier coverage application is to detect invaders when they cross a border or penetrate a protected area. Barrier coverage guards boundaries of critical assets. Examples of such boundaries are country borders, marginal lines and boundaries of battlegrounds. Some sensor deployment methods and the crossing routes taken by the invader affect the barrier coverage. One method is deploying the sensors in regions of non uniform long belt form. Another method is to manually place in desired locations.

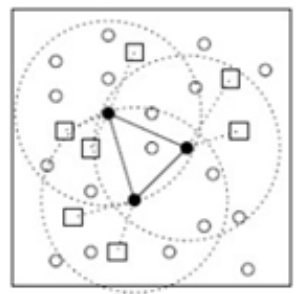

(b)

Fig. 4. Point Coverage

Another method is to randomly deploy such as dropped by aircrafts or started by artilleries. Barrier coverage calculates the motion of objects in the selected area. Movement rate of objects and sensing power of the sensors determine the chances of probability for every point on the way that the object follows. The density of sensor nodes can be computed with this calculation. Barrier coverage is of two types: - Weak k-barrier coverage and Strong k-barrier coverage.

In weak barrier coverage, invaders moving through consistent routes are detected and in strong barrier coverage whatever crossing paths the intruders take, they are detected.

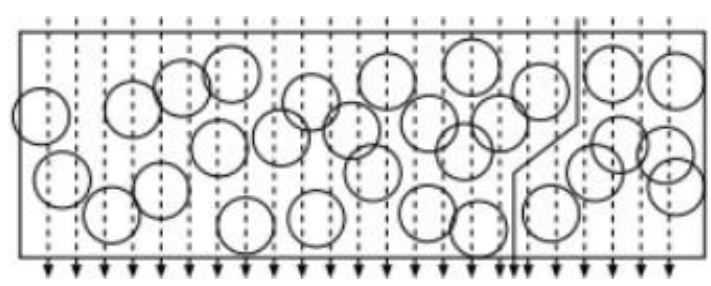

Fig. 5. Weak k-barrier coverage

\section{B. Network Deployment}

iStatic Network iiRandom Network

1) Static Network: In static coverage, the form of the network and position of sensor nodes are known earlier. Sensor nodes are located in a predetermined pattern in static coverage. But in many cases deployment of sensor nodes in deterministic pattern is impossible. An example of static coverage is the art gallery problem [23] .

For instance, in static coverage, sensors are deployed at equal distances. In fig.6 the horizontally deployed sensors are at equal distance from each other and the distance between 
the vertically placed sensors are equal. Assume the distance between the horizontally placed 1 st sensor to 2 nd sensor is $1 \mathrm{~cm}$ then the distance between the horizontally placed $2 \mathrm{nd}$ sensor to $3 \mathrm{rd}$ sensor is also $1 \mathrm{~cm}$. And the distance between vertically placed sensors is $2 \mathrm{~cm}$. So, every distance of the vertical sensors are $2 \mathrm{~cm}$.

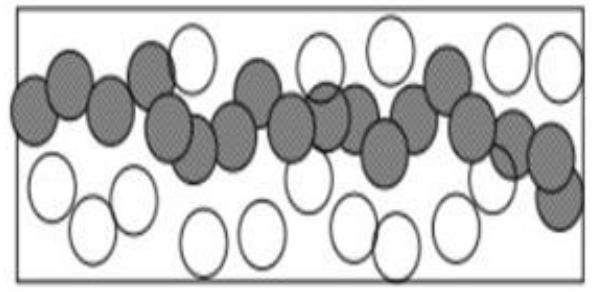

Fig. 6. Strong k-barrier coverage

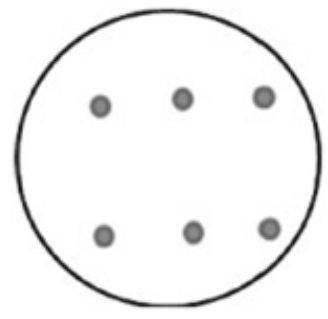

Fig. 7. Static deployment

2) Random Network: The opposite of static network is the random network. Random network means position of sensors and network's topological shape is not known, there is no predefined information about it. In random network, considering the nodes as stationary, nodes is placed very close to achieve desired coverage. Thus, to maintain coverage while minimizing energy consumption is the aim of random network. In random coverage, the sensors are deployed randomly, and the distance between the sensors are not equal neither horizontally nor vertically.

\section{Node Sensing Models}

iBinary Model iiProbabilistic Model

Binary Model: Binary Model is the simplest model. In this model, a disc shaped region is sensed by the node. In this model, a node has the ability of sensing from the points that is inside the sensing range. The points beyond the sensing range cannot be sensed by the node. In binary model, sensing range of all nodes is known as sensing radius is a circular disk of radius $r$.

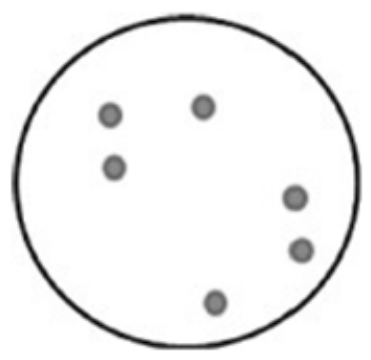

Fig. 8. Random deployment

$$
C_{x y}\left(S_{i}\right)= \begin{cases}1, & \text { if } d\left(S_{i}, p\right)<r \\ 0, & \text { otherwise }\end{cases}
$$

2) Probabilistic Model: Probabilistic model is continuation of the previous sensing model. In this model [18] $\mathrm{Ru}<\mathrm{Rs}$ where $\mathrm{Ru}$ is a quantity, $\mathrm{Rs}$ is the radius of round-shaped disk and ( $\mathrm{Rs} \mathrm{Ru}, \mathrm{Rs}+\mathrm{Ru}$ ) is defined, and a probability of an object to be detected is ' $p$ '. According to the probabilistic model, a sensor that senses a point $\mathrm{p}(\mathrm{x}, \mathrm{y})$ is given as:

$$
C_{x y}\left(S_{i}\right)= \begin{cases}0, & \text { if } R_{s}+R_{u}<=d\left(S_{i}, p\right) \\ e^{-\lambda \alpha^{\beta}}, & \text { if } R_{s}-R_{u}<d\left(S_{i}, p\right)<R_{s}+R_{u} \\ 1, & \text { if } R_{S}-R_{u}>=d\left(S_{i}, p\right)\end{cases}
$$

where, $\alpha=\mathrm{d}(\mathrm{Si}, \mathrm{p}) \quad(\mathrm{Rs} \mathrm{Ru})$, and $\lambda, \beta$ are parameters that compute detection probability.

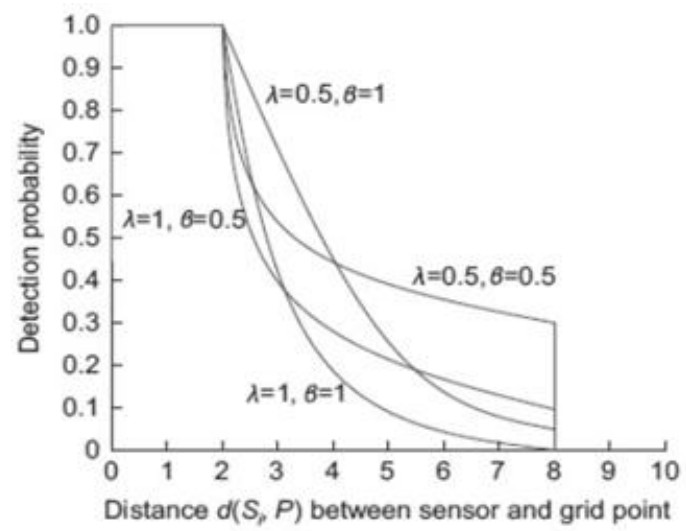

Fig. 9. Probabilistic sensor detection model

Different values of the parameters $\lambda$ and $\beta$ yield different translations by different detection probabilities.

\section{Target Characteristics}

\section{iStatic Target Coverage iiDynamic Target Coverage}

1) Static Target Coverage: The targets that are not capable of moving and fixed relating to sensing node are called static targets, also known as stationary targets. The goal of static target coverage is to increase coverage and decrease redundancy of sensor nodes. As seen in day to day life monitoring of temperature is an example of this and is the simplest approach. Entrance of a university can be the case of static target.

2) Dynamic Target Coverage: The targets that can move and are mobile to the corresponding sensing node are called dynamic

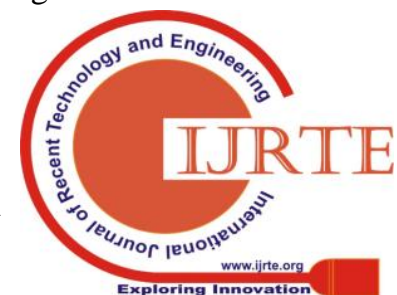


targets. The movement of the dynamic target is the main part of this type of coverage. Compared to static target, dynamic target is much more complicated. In case of military purposes to use for the purpose of battlefield surveillance dynamic target coverage is very useful. A running car, a moving wheel can be considered as an example of dynamic target.

\section{E. Application Attributes}

iEnergy-Saving Coverage iiConnectivity Coverage

2) Energy-Saving Coverage: The work of energy-saving coverage is 'the energy utilization by the nodes in the network is in a systematic way or not' as it has an effect on whether the network exists. This type of coverage is done by separating the sensing nodes into groups of "active" and "sleep" nodes in different stages as it has limited resources. 1) Connectivity Coverage: Connectivity coverage can con-

trol both communication necessity and level of coverage at the same moment.

Connectivity coverage is categorized as-

a) Active role connectivity coverage. b)Connected path coverage.

Active role connectivity coverage is concerned with the "work- ing" and "sleep" condition of nodes in rotation.

Connected path coverage is concerned with the selection process of attached sensor nodes to obtain maximal impact.

\section{COVERAGE TECHNIQUES}

In this section different coverage techniques are defined to get maximum efficient coverage. There are two coverage techniques- Static coverage and dynamic coverage. At first we describe the various static coverage of Wireless sensor network.

\section{A. Efficient Coverage Area}

1) Deployment of sensor based on Equilateral triangle: Theroem: If three circles $\mathrm{C} 1, \mathrm{C} 2, \mathrm{C} 3$ encircle three seamless topology disks D1,D2,D3 which intersect at a point then $\mathrm{C} 1 \mathrm{C} 2 \mathrm{C} 3$ is an equilateral triangle and the seamless topology area is maximum and its value is and the coverage efficiency is $94.2 \%$.

2) Hypothesis

Some theoretical hypothesis is:

Hypothesis1: A sensor's sensing capability is its coverage extent which is a circle where radius is ' $r$ ' and area is ' $D$ '= $\pi \mathrm{r} 2$.
Hypothesis2: All sensor's radio power is uniform in a sensor area.

Hypothesis3: All sensor's lie in the same flat surface (level) in a sensor area.

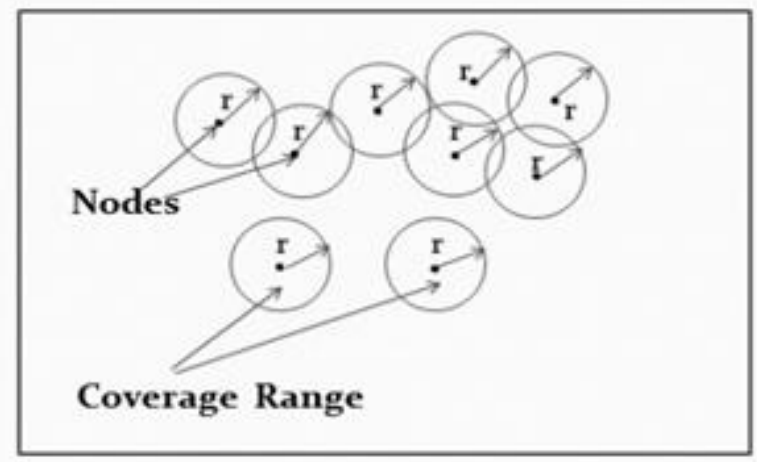

Fig. 10. Sensors coverage extent and sensor area of WSN

2) Definations

Definition 1: Efficient coverage area denoted as SECA is the coverage area that is node A1's coverage range subtracted from the overlapping coverage zone Z's area denoted as in [1].

$$
\begin{aligned}
S_{E C A} & =D-S_{Z} \\
S_{E C A} & =\pi r^{2}-S_{Z}
\end{aligned}
$$

Definition2: Efficient coverage area ratio is $R_{E C A}=$ $\frac{S_{E C A}}{D}$

$$
\begin{aligned}
& R_{E C A}=\frac{\left(D-S_{Z}\right)}{D} \\
& R_{E C A}=1-\frac{S_{Z}}{D} \\
& R_{E C A}=1-\frac{S_{Z}}{\pi r^{2}}
\end{aligned}
$$

In fig. 12, for circle $\mathrm{C} 2$ and $\mathrm{C} 3$ point $\mathrm{A}$ and point $\mathrm{B}$ are the intersecting points and a point in circle $\mathrm{C} 1$ is at least between point $\mathrm{A}$ and point $\mathrm{B}$. To realize full seamless coverage as shown in fig 13 , only if circle $\mathrm{C} 1$ is through point $\mathrm{A}$ and point $\mathrm{B}$ and centre $\mathrm{C} 1$ is outside disk D2 and disk D3 the efficient coverage area of the three disks D1, D2, D3 is possible.

The gray area in fig.14 is minimum if the efficient coverage area in fig. 13 be maximum. And if the gray area of fig. 14 be minimum then let the segment of $\mathrm{C} 1 \mathrm{C} 2, \mathrm{C} 2 \mathrm{C} 3$, and $\mathrm{C} 3 \mathrm{C} 1$ or let the centre $\mathrm{C} 1, \mathrm{C} 2, \mathrm{C} 3$ be maximum which means on the condition of full and seamless coverage the area of $\mathrm{C} 1 \mathrm{C} 2 \mathrm{C} 3$ be maximum. 


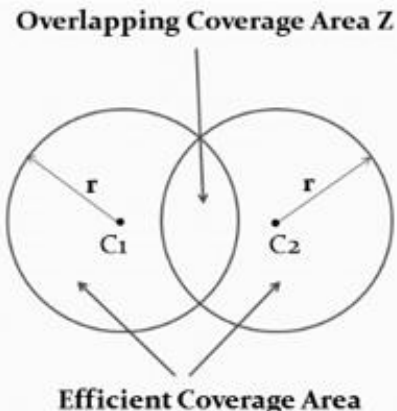

Fig. 11. Efficient Coverage Area

As shown in fig.15, $\mathrm{A}$ is centre, point $\mathrm{C} 1, \mathrm{C} 2, \mathrm{C} 3$ are concyclic. In fig. 15, an arbitrary point $\mathrm{C} 1$ ' of circle $\mathrm{A}$ apart from point $\mathrm{C} 1$ is taken and an auxiliary $\mathrm{C} 1{ }^{\prime} \mathrm{C} 2 \mathrm{C} 3$ is drawn. In $\mathrm{C} 1$ ' $\mathrm{C} 2 \mathrm{C} 3$, of edge $\mathrm{C} 2 \mathrm{C} 3 \mathrm{C} 1$ ' $\mathrm{P} 2$ is the perpendicular. And in $\mathrm{C} 1 \mathrm{C} 2 \mathrm{C} 3$, of edge $\mathrm{C} 2 \mathrm{C} 3, \mathrm{C} 1 \mathrm{P} 1$ is the perpendicular and is through centre A. And from the fig.15), it is seen that $\mathrm{C} 1 \mathrm{C} 2 \mathrm{C} 3$ is an equilateral triangle.

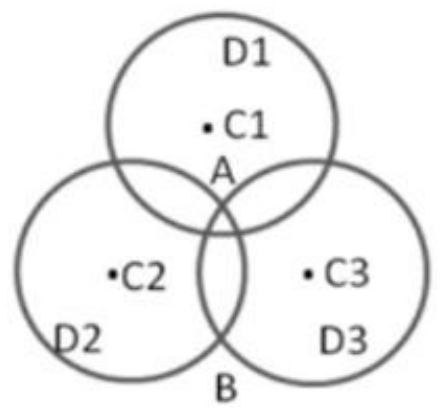

Fig. 12.

Now in fig.18, the shaded area $\mathrm{M} 1=$ the area of the sector AC2A'-the area of O AC2P3

$$
\begin{aligned}
& =\frac{1}{2} r^{2} \theta-\frac{1}{2} * \text { base } * \text { height } \\
= & \frac{30^{\circ} r^{2}}{2}-\frac{1}{2} *|C 2 P 3| *|A P 3| \\
= & \frac{30^{\circ} r^{2}}{360^{\circ}}-\frac{1}{2} * \sqrt{r^{2}-\left(\frac{r}{2}\right)^{2}} * \frac{r}{2} \\
= & \frac{\pi r^{2}}{12}-\frac{r}{4} * \sqrt{\frac{3 r^{2}}{4}} \\
= & \frac{\pi r^{2}}{12}-\frac{r^{2} \sqrt{3}}{8}
\end{aligned}
$$

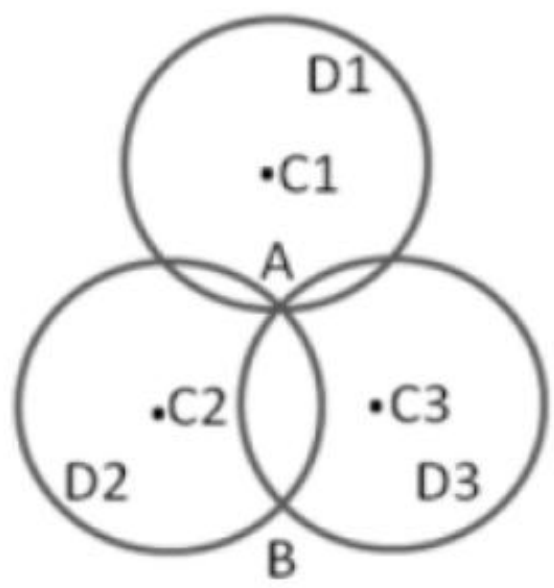

Fig. 13.

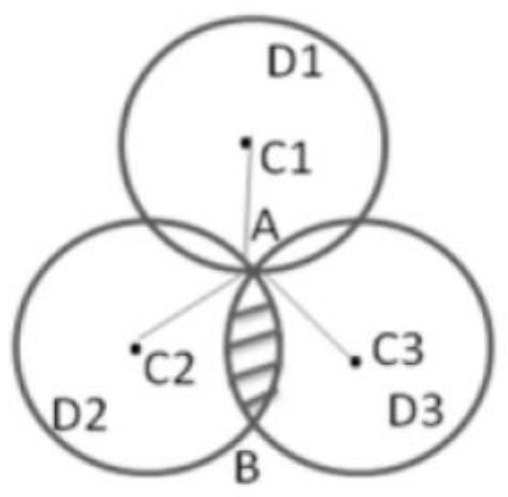

Fig. 14.

$$
=\frac{2 \pi r^{2}-3 \sqrt{3} r^{2}}{24}
$$

In fig. 19 because of equality, the shaded area M2 $=4 * M 1$ $=4 * \frac{2 \pi-3 \sqrt{3}}{24} r^{2}$

$$
=\frac{2 \pi-3 \sqrt{3}}{6} r^{2}
$$

In fig. 20 because of equality, the shaded area M3 $=2 * M 2$ $=2 * \frac{2 \pi-3 \sqrt{3}}{6} r^{2}$

$$
=\frac{2 \pi-3 \sqrt{3}}{3} r^{2}
$$

In fig. 21 the shaded area M4=D $-M 3$

$=\pi r^{2}-\frac{2 \pi-3 \sqrt{3}}{3} r^{2}$
$=\frac{3 \pi r^{2}-2 \pi r^{2}+3 \sqrt{3} r^{2}}{3}$

$$
=\frac{\pi+3 \sqrt{3}}{3} r^{2}
$$

In fig.22 the gray area M5 $=3 * M 4+3 * M 2$ $=3 * \frac{\pi+3 \sqrt{3}}{3} r^{2}+3 * \frac{2 \pi-3 \sqrt{3}}{6} r^{2}$ 

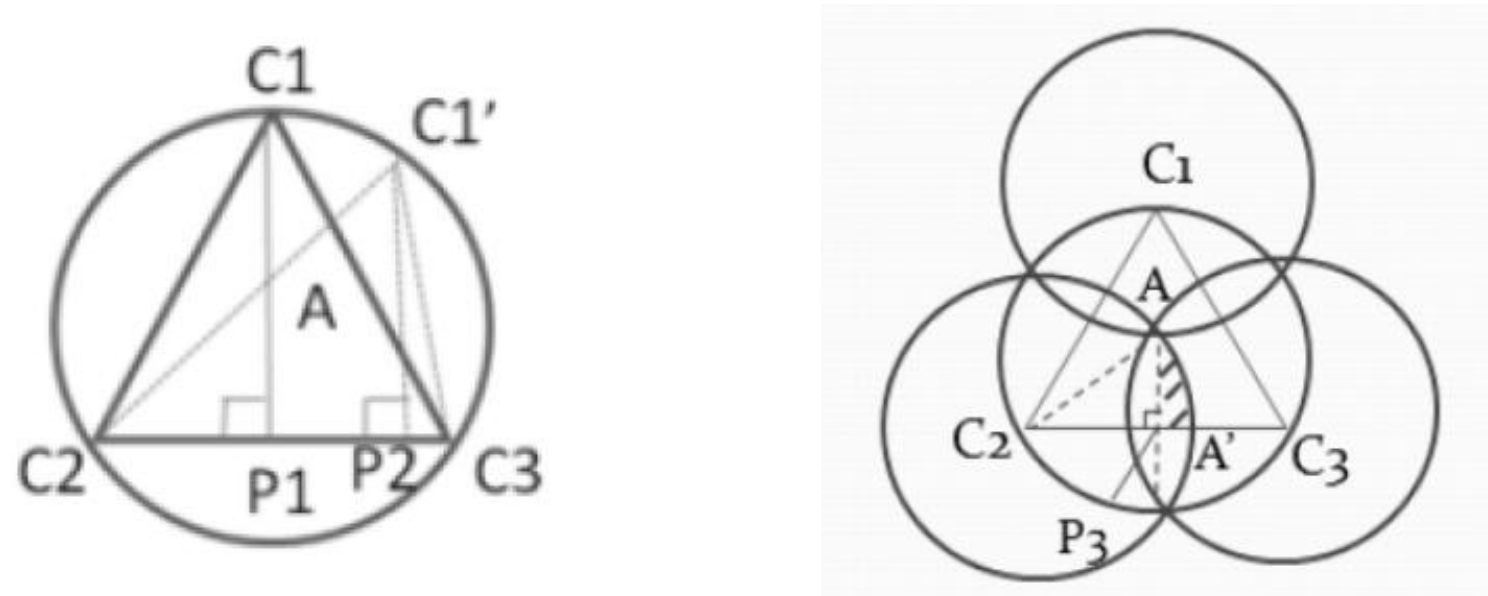

Fig. 18.

Fig. 15.

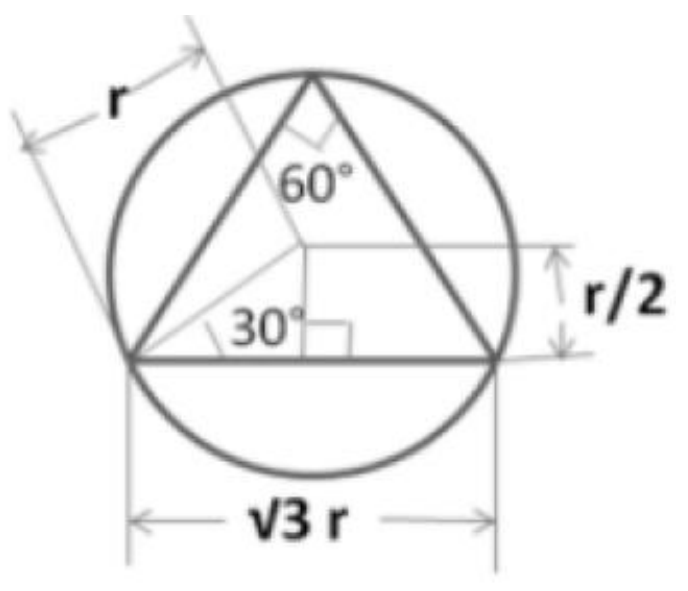

Fig. 16.

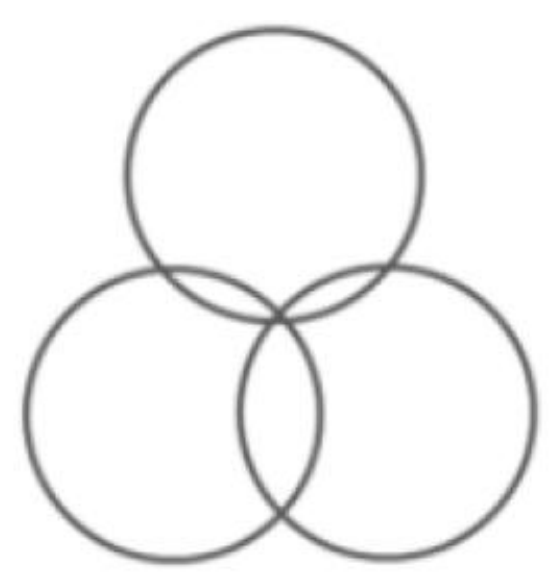

Fig. 17.

$$
\begin{aligned}
=\frac{2 \pi r^{2}+6 \sqrt{3} r^{2}+2 \pi r^{2}-3 \sqrt{3} r^{2}}{2} & \\
= & \frac{4 \pi+3 \sqrt{3}}{2} r^{2}
\end{aligned}
$$

For r $=1, S_{E C A t}=8.8813$

Now, the efficient coverage area, $R_{E C A t}=\frac{S_{E C A t}}{3 \pi r^{2}}$

$$
\begin{aligned}
& =\frac{\frac{(4 \pi+6 \sqrt{3}) r^{2}}{2}}{3 \pi r^{2}} \\
= & \frac{(4 \pi+6 \sqrt{3}) r^{2}}{6 \pi r^{2}} \\
= & 0.942
\end{aligned}
$$

Therefore, the coverage efficiency is $94.2 \%$

Therefore, the coverage efficiency is $94.2 \%$

2) Deployment of sensors based on Hexagonal mesh: The circle $\mathrm{C} 3$ at the centre intersects with six peripheral circles at six points or centre's of each of the six circles which divide the central circle into six equal parts. Thus a hexagon is formed. Now obviously, the edge length of the hexagon is equal to radius $r$. Now to find the area of the central circle, first we find the overlapping areas of the central circles then we subtract overlapping area from the whole area denoted as $D$, From the above solved equations as in (7) we get the area of the shaded overlapping area which is, 


$$
\frac{2 \pi-3 \sqrt{ } 3}{6} r^{2}
$$

Therefore, the area of six overlapping parts is,

$=\frac{2 \pi-3 \sqrt{3}}{6} r^{2} * 6$

$$
=(2 \pi-3 \sqrt{3}) r^{2}
$$

Now, the efficient coverage area of the central circle is,

$=D$ - overlappingarea

$=D-(2 \pi-3 \sqrt{3}) r^{2}=\pi r^{2}-2 \pi r^{2}+3 \sqrt{3} r^{2}$

$$
(-\pi+3 \sqrt{3}) r^{2}
$$

We know, the area of the circle is $\pi r^{2}$

Proof - The sum of the overlapping area and the efficient coverage area should be equal to $\pi r^{2}$.

That is, $(2 \pi-3 \sqrt{3}) r^{2}+(-\pi+3 \sqrt{3}) r^{2}$

$=2 \pi r^{2}-3 \sqrt{3} r^{2}-\pi r^{2}+3 \sqrt{3} r^{2}$

$=\pi r^{2}$

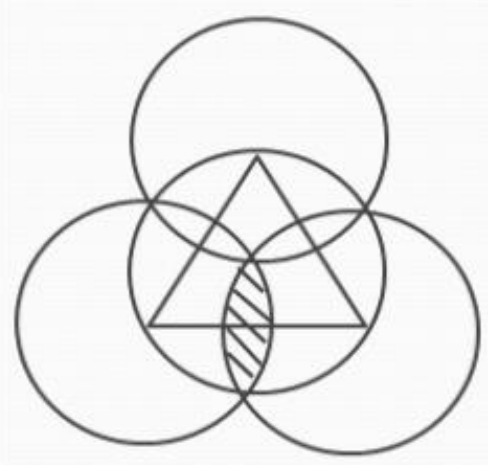

Fig. 19.

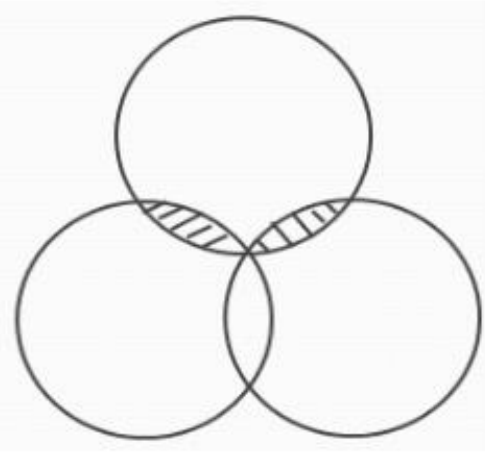

Fig. 20

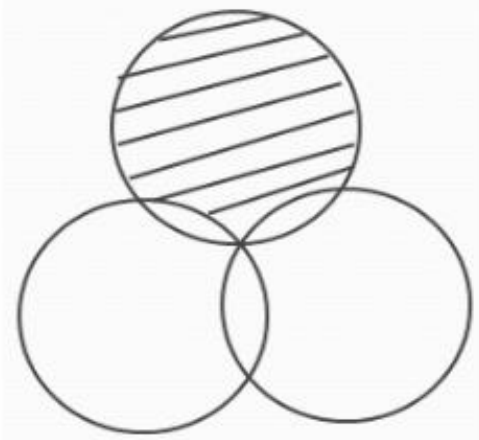

Fig. 21

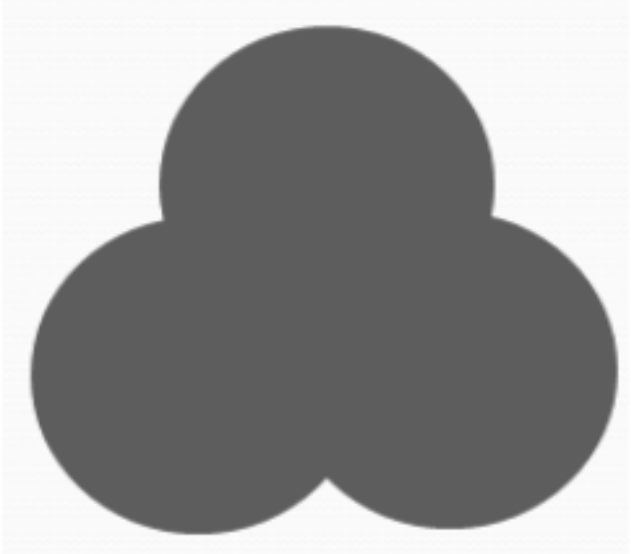

Fig. 22.

Now, the maximum efficient coverage area for hexagonal mesh is $4 \pi+63 \mathrm{r} 2$.

And the coverage efficiency is $90.2 \%$.

3) Deployment of sensors based on Square Mesh: As shown in Fig.24, four circles intersect at the point at which the two diagonals of the square intersect. Therefore, the shaded overlapping area of the circle is,

$$
=\frac{4 \pi-6 \sqrt{3}}{3} r^{2}
$$

Now, the efficient coverage area of that circle is = Doverlapping area

$=\pi r^{2}-\frac{4 \pi-6 \sqrt{3}}{3} r^{2}$
$=\frac{3 \pi r^{2}-4 \pi r^{2}+6 \sqrt{3} r^{2}}{3}$

$$
=\frac{(-\pi+6 \sqrt{3})}{3} r^{2}
$$

We know, the area of a circle is $\pi r^{2}$.

Proof- The four overlapping area plus the efficient coverage area should be equal to $\pi r^{2}$.

That is, $\frac{4 \pi-6 \sqrt{3}}{3} r^{2}+\frac{(-\pi+6 \sqrt{3})}{3} r^{2}$

$=\frac{4 \pi r^{2}-6 \sqrt{3} r^{2}-\pi r^{2}+6 \sqrt{3} r^{2}}{3}$

$=\frac{3 \pi r^{2}}{3}$
$=\pi r^{2}$

Now, the maximum efficient coverage area for square mesh is $\left(\frac{(\pi+2)}{2 \pi}\right) r^{2}$

And the coverage efficiency is $81.8 \%$.

4) Deployment of sensors based on Octagonal Mesh: For six topology disks of six circles which are divided at a point, a hexagon which is formed by the midpoints of the six circles is a uniform hexagon. Considering circle $\mathrm{C} 1$ from the fig.25, the one overlapping area of that circle which is the part of the hexagon is,

$=\frac{2 \pi-3 \sqrt{3}}{6} r^{2}$

Now the seven overlapping areas of the circle is, 


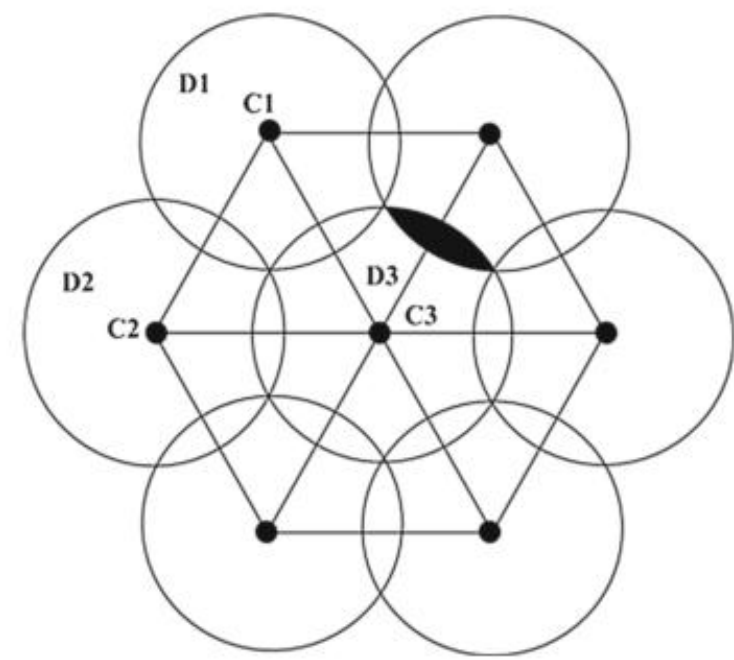

Fig. 23. Hexagonal Mesh

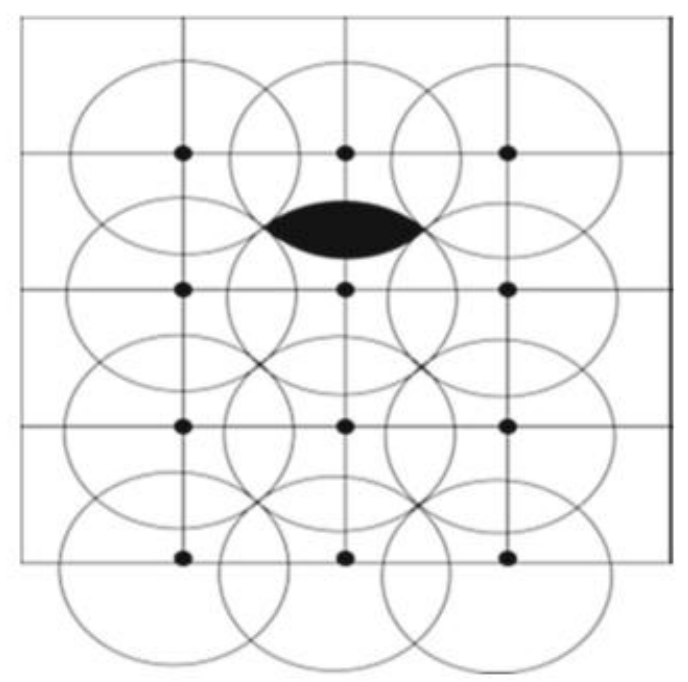

Fig. 24. Square Mesh

$$
\begin{aligned}
& =\frac{2 \pi-3 \sqrt{3}}{6} r^{2} * 7 \\
& \frac{14 \pi-21 \sqrt{3}}{6} r^{2}
\end{aligned}
$$

Therefore, the efficient coverage area of the circle is, =D overlapping area

$=\pi r^{2}-\frac{14 \pi-21 \sqrt{3}}{6} r^{2}$

$=\frac{6 \pi r^{2}-14 \pi r^{2}+21 \sqrt{3} r^{2}}{6}$

$=\frac{-8 \pi r^{2}+21 \sqrt{3} r^{2}}{6}$

$$
\frac{-8 \pi+21 \sqrt{3}}{6} r^{2}
$$

We know, the area of a circle is $\pi r^{2}$.

Proof- The sum of overlapping area and the efficient coverage area should be equal to $\pi r^{2}$.

$=\frac{14 \pi-21 \sqrt{3}}{6} r^{2}+\frac{-8 \pi+21 \sqrt{3}}{6} r^{2}$

$=\frac{14 \pi r^{2}-21 \sqrt{3} r^{2}-8 \pi r^{2}+21 \sqrt{3} r^{2}}{6}$

$=\frac{6 \pi r^{2}}{6}$

$=\pi r^{2}$

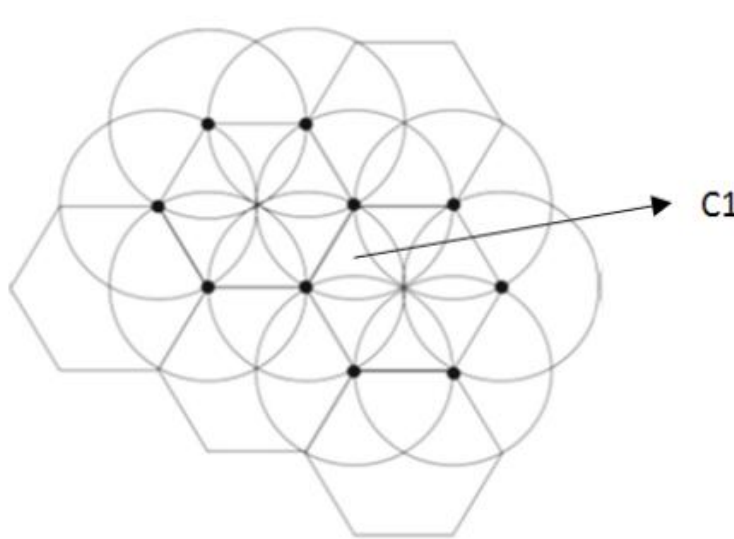

Fig. 25. Octagonal Mesh

Therefore, the maximum efficient coverage area for honeycomb mesh is $2 \pi+3 \sqrt{ } 3 \mathrm{r} 2$

And the coverage efficiency is $60.9 \%$.

\section{A. Path Coverage}

In path coverage, nodes are located to sense a route and if any intruder tries to cross it then it reports. In a manual deployed network, if sensors are placed properly over the area then the desired path coverage is found. In case where manual deployment is not possible random deployment by [7] [8] [9]is used. But there is no guaranty of the desired full path coverage in random deployment. In [4] [5] [6] has worked mainly on path coverage by random network.

\section{B. $K$-coverage}

The aim of k-coverage problem is to determine whether every point in the area is covered by at least $\mathrm{k}$ sensors where $\mathrm{k}$ is a predetermined value. Finding out all sub regions divided by the sensing boundaries of all $\mathrm{n}$ sensors and then checking if each sub region is $\mathrm{k}$ covered or not is one solution. $\mathrm{K}$-coverage can be $1,2,3 \ldots \ldots \mathrm{n}$ covered. When two sensors intersect each other, the overlapping portion is 2-covered, whereas the remaining portions of the sensors are 1-covered. Similarly, more than two sensors can intersect each other and 3-covered 4- covered cases may occur. When the sensors are tangent to each other that means there is no connectivity. When sensors are not intersecting then there may be loss of connectivity.

- 1-covered

- 2-covered

- 3-covered

C. Graph Based 
In Computational geometry, Voronoi diagram as shown in

[10] is an important data structure. Voronoi diagram is set of distinct points and represents the proximity information about a set of geometric nodes. Voronoi diagram partitions each flat surface into a number of polygons in a manner that all points are closest to any one point.

The attributes of electromagnetic particles motivates an algorithm named Vector based algorithm described by [11]. An expelling force pushes two electro-magnetic particles apart when they come too close to each other. Assume the distance between sensor $\mathrm{Si}$ and sensor $\mathrm{Sj}$ is dij. The average distance between two sensors is dave when the sensors are evenly distributed in the target area. A distance of (dave $\mathrm{dij}$ )/2 is pushed to move away from each other between the sensors $\mathrm{Si}$ and $\mathrm{Sj}$ by the virtual force between the two sensors $\mathrm{Si}$ and $\mathrm{Sj}$. In case when one sensor should not move and covers its Voronoi polygon completely, the other sensor will be pushed dave dij away. If coverage hole exists in either of their Voronoi polygons, the virtual force will push the sensors dave away from each other.

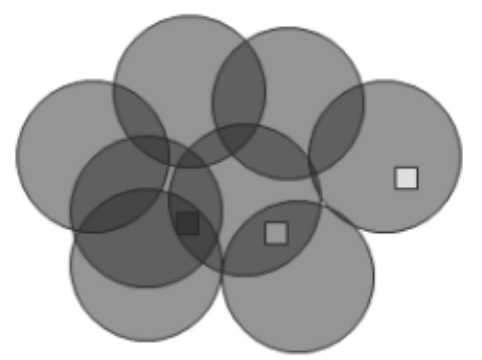

Fig. 26. An example of 1-covered, 2-covered and 3-covered

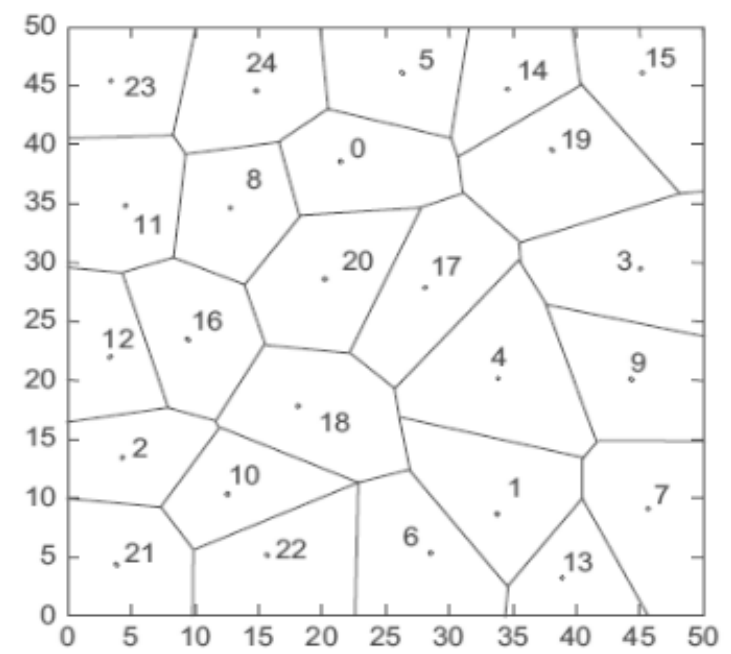

Fig. 27. Voronoi diagram

D. Repair policies of coverage hole
Sensors are placed arbitrarily in a region, and no extra nodes are allowed in the network. Because of destruction of a node or the active node count exceeds margin, a coverage gap is noticed in the network then the network may be enlarged by extra nodes.

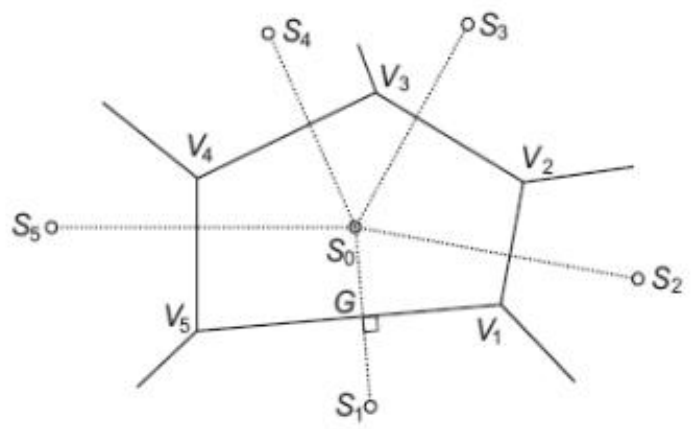

Fig. 28. Voronoi Polygon of So

The damage of a node because of failure affects the network coverage and network connectivity. Maintaining network con- nectivity when a node fails while preserving the pre failure coverage is the main work of repair policies. An example of network topology is illustrated in fig. 29. In [13], there are 13 nodes where nodes $\mathrm{n} 1, \mathrm{n} 2, \mathrm{n} 3, \mathrm{n} 10$ and $\mathrm{n} 11$ are neighbors of $n 9$. If node $n 9$ fails, it would disconnect nodes $\mathrm{n} 10, \mathrm{n} 11$ and their neighbors from the remaining of the network and leave a coverage gap as no other nodes sensing range is overlapping with $n$ 9. If failed node $n 9$ is replaced with other node the connectivity will be restored and the coverage hole is only shifted to another place either in the inner part or at the ends.

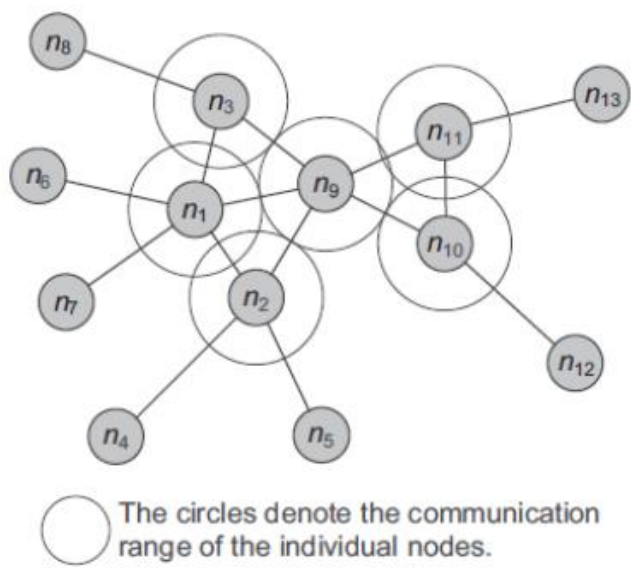

Fig. 29. Selection of mobile sensor nodes rooted on hop count

Coverage Conscious Connectivity Restoration (C3R) algorithm is the algorithm that overcomes the problem. It 
temporarily replaces the unsuccessful node by one or more of its nearest nodes. The nodes shift in order to maintain coverage and network topology.

In $\mathrm{C} 3 \mathrm{R}$ algorithm, $\mathrm{F}$ is a failed node and $\mathrm{A}$ is the node that replaces F. As mentioned earlier failure of node $\mathrm{F}$ causes a hole in the network coverage. If node A replaces node $F$ then the coverage hole shifts from one location to another. In C3R, the reallocated node moves back and forth between $\mathrm{F}$ and its original location and it also does not settle at the position of $\mathrm{F}$. Thus, temporary coverage and irregular connectivity is provided by C3R to localize the extent of recovery and avoid a full loss of coverage. In C3R algorithm, the neighbor nodes of the failed node $\mathrm{F}$ takes the place of the unsuccessful node and serve for some time, then it will go back to its original position allowing other neighbors of $\mathrm{F}$ to come and serve.

Another distributed algorithm proposed in [14] uses laws of vectors as a tool to decide the magnitude and direction of the mobile nodes that recovers the holes. Here mobility of nodes is limited by only one hop as mobility of nodes consumes more energy. In fig.30 an example of detecting flexibility of nodes is shown.

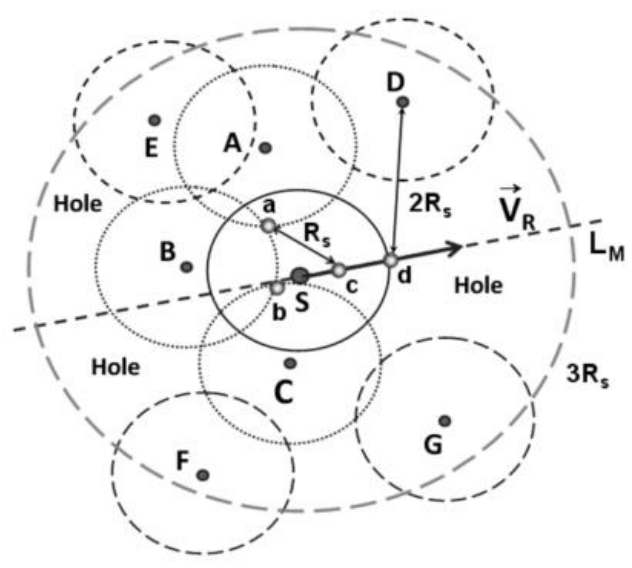

Fig. 30. Example of detecting mobility distance of active node $\mathrm{S}$

$\mathrm{Si}$ and $\mathrm{Sj}$ are two given nodes, if $0<\mathrm{dij}<2 \mathrm{Rs}$, then $\mathrm{Si}$ and $\mathrm{Sj}$ intersect each other and is called close-workers. Now if 2Rs < $\mathrm{dij}<3 \mathrm{Rs}$, then $\mathrm{Si}$ and $\mathrm{Sj}$ is apart from each other and is called co-workers where dij is the physical distance between $\mathrm{Si}$ and $\mathrm{Sj}$. Let $\mathrm{S}$ be a mobile node that calculate its mobility distance. Let A, B and C be the close-workers of S and D, E, F and G be co-workers. The direction of the resultant vector denoted by $\mathrm{V}^{*} \mathrm{R}$ is found by a algorithm called resultant vector construction algorithm. ' $a$ ' and ' $b$ ' are intersection points at node $\mathrm{S}$. Let $\mathrm{LM}$ be the straight line along $\mathrm{V}^{\cdot} \mathrm{R}$ and passes through position node $\mathrm{S}$. Let ' $\mathrm{a}$ ' be the intersection point from which distance between $S$ and ' $a$ ' is longest compared to the distance between $\mathrm{S}$ and $\mathrm{b}$. So from a, a point c is taken on straight line LM such that ac = Rs units.
Similarly, the co- worker of $\mathrm{S}$ closest to it is found. Let co-worker $\mathrm{D}$ be the closest. Now from node $\mathrm{D}$, a point $\mathrm{d}$ is taken on LM such that $\mathrm{Dd}=2 \mathrm{Rs}$ units. Now, the minimum value between $\mathrm{Sc}$ and $\mathrm{Sd}$ is calculated and the minimum one is considered to be mobility distance of $\mathrm{S}$.

\section{A. Virtual force}

Deployment of sensors randomly in the aimed area is considered as the first deployment scheme if there is no prior knowledge. In military applications, random deployment is practical where all sensors are dropped or thrown in the sensor area. The virtual force algorithm (VFA) works as a sensor deployment scheme to increase the coverage when initially the sensors are randomly placed. Let Fi be the total force action on sensor $\mathrm{Si}$, where $\mathrm{Fi}$ is a vector whose orientation is determined by the vector sum of all the forces acting on Si. Let Fij be the force exerted by another sensor $\mathrm{Sj}$ on $\mathrm{Si}$. A sensor $\mathrm{Si}$ is directed to forces exerted by obstacles.

Here, let $\mathrm{F}^{*} \mathrm{~A}$ be the total force of attraction on $\mathrm{Si}$ due to preferential coverage areas. LetF $\mathrm{R}$ be the total force of repulsive on $\mathrm{Si}$ due to obstacles. Now, the total force Fi on Si can be expressed as

$$
\overrightarrow{F_{i, j}}=\sum_{j=1, j \neq i}^{k} \overrightarrow{F_{i, j}}+\overrightarrow{F_{R}}+\overrightarrow{F_{A}}
$$

Next, the force $\mathrm{Fi}, \mathrm{j}$ between $\mathrm{Si}$ and $\mathrm{Sj}$ in polar form is

expressed. A magnitude of $\gamma$ and orientation $\theta$ for vector $f$ is implied as $\mathrm{f}=(\gamma, \theta)$

$$
F_{i j}= \begin{cases}\left(W_{A}\left(d_{i j}-d_{t h}\right), \alpha_{i j}\right), & \text { if } d_{i j}>d_{t h} \\ 0, & \text { if } d_{i j}=d_{t h} \\ \left(W_{R} \frac{1}{d_{i j}}, \alpha_{i j}+\pi\right), & \text { if } d_{i j}<d_{t h}\end{cases}
$$

Where,

dij $=$ Euclidean distance between sensor $\mathrm{Si}$ and $\mathrm{Sj}$.

$\mathrm{dth}=$ Threshold on the distance between sensor $\mathrm{Si}$ and $\mathrm{Sj}$.

$\alpha i j=$ Angle of a line segment from $\mathrm{Si}$ to $\mathrm{Sj}$.

$\mathrm{WA}=\mathrm{A}$ measure of the attractive force

$\mathrm{WR}=\mathrm{A}$ measure of the repulsive force.

How close sensors can get to each other is controlled by the Threshold distance dth. The regional coverage after first random deployment can be 
increased by sensor deployment technique based on virtual forces is shown in the simulation result of [15].

The improved shape of the virtual force rooted tech- nique with extra concern of the connectivity necessity is Connectivity-Preserved Virtual Force (CPVF) strategy described elaborately in [16]. This scheme guarantees connectiv- ity for a network with random sensor extents and maximizes sensing coverage. Any information about the field layout which can be irregular and having holes of arbitrary shape is not needed in this scheme.

\section{SLEeP SChEdUling MechanisM}

In WSN application energy is concerned which needs to perform for a huge period with the insufficient battery capacity. For power preservation in WSN an approach is arranging sleep breaks for extra nodes while to provide continuous service the remaining nodes stay active. Successful operation for the sensor network is possible if the active nodes maintain both network connectivity and sensing coverage.

For better understanding of sleep scheduling mechanisms, the different power saving modes given by a sensor are needed to be differentiated. The crucial modes of a sensor are

iOff-duty iiTransceiver On-duty iiiSensing unit On-duty ivOn-duty

Off-duty: The other name of this mode is sleep mode. Here, a timer or a triggering mechanism may be running to wake up a sensor while each and every elements of a sensor are switched off.

Transceiver On-duty: In this mode, transmitting, receiving and processing is done by the sensor but not sensing. The processor and transceiver are turned on but all the sensing units are turned off.

Sensing unit On-duty: In this mode, sensing and processing sensory data are done by the sensor. Transmitting or receiving of messages is not done. The processor and at least one sensing unit are turned on but the transceiver is turned off.

On-duty: The other name of this mode is active mode. Here the sensor does sending or receiving of messages, collects sensory data, process data and messages. In this mode, a sensor's all components are turned on.

\section{A. Self-scheduling strategy}

Self-scheduling strategy makes needless nodes in an off- duty condition. All the nodes that are deployed from that only the maximum number of active nodes is selected by selfscheduling strategy to do sensing task for guarantying enough coverage of the observed area while each individual sensor node's energy consumption is reduced to extend network duration as discussed by [17].

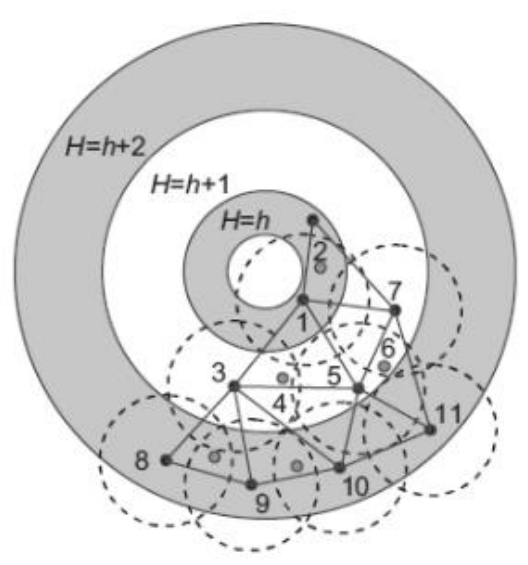

Fig. 31. Example of choosing mobile sensor nodes rooted on hop count information.

In [18], a more realistic sleep scheduling algorithm is suggested to extend the whole lifetime of a network. The another name of this algorithm is Connected $\mathrm{K}-$ Neighborhood $(\mathrm{CKN})$. CKN is a distributed algorithm and it turn off the needless nodes and at the same time the network satisfies $\mathrm{k}$ - connectivity. If more than $\mathrm{k}$ neighbors are present in a node than at least $\mathrm{k}$ of them remain awake while the others goes to sleep. And if a node has less than $\mathrm{k}$ neighbors than every neighbor remains awake, none of them goes to sleep. The active nodes are chosen totally based on the ranks by the CKN algorithm. The ranks are given randomly at the starting of the execution of the $\mathrm{CKN}$ algorithm in each time period. The set of waken nodes transforms time to time. It is due to $\mathrm{CKN}$ algorithm that $\mathrm{CKN}$ cannot guaranty power utilization consistently.

\section{ADJUSTABLE COVERAGE RADIUS}

The sensing extent of a sensor is definite is assumed by many networks. Another power saving techniques is adjusting the transmission or sensing range of the wireless sensor nodes. For solving target coverage problems, radius adaptive method is used. Decreasing the overlaps between sensing ranges and maintaining the quality of service of coverage over predetermined identification degree is the major goal of radius adaptive mechanism.

\section{A. Target Coverage}


Assume, to cover $\mathrm{s}$ targets there are $\mathrm{n}$ sensors that are randomly deployed.

Definition of Target Coverage.

With $\mathrm{N}$ sensors deployed randomly in the targets area where $\mathrm{M}$ is the given target with known position and a power constrained WSN, arrange the sensor nodes action such that all targets are constantly noticed and network lifespan is expanded. Here the approach used by [19] is to arrange the sensors in groups where one group is responsible for observing the targets while all other sensors are in sleep mode. In this approach locating the sensing range of all active sensors is concerned along with determining the set covers. The aim is to meet the target requirement and apply least sensing range for decreasing the power utilization.

The Adjustable Range Set Covers problem [20] is applied to solve the target coverage issue.

\section{B. Definition of Adjustable Range Set Covers Problem.}

Finding a family of set covers $\mathrm{c} 1, \mathrm{c} 2, \ldots$. ..ck where a set of sensors with adjustable sensing ranges and a set of targets are given and determining the sensing range of each sensor in each set such that $\mathrm{K}$ is maximized, each sensor set monitors all targets and each sensor appearing in the sets $\mathrm{c} 1, \mathrm{c} 2$, . . ..ck consumes at most energy E.

An example with four sensors s1, s2, s3, s4 and three targets $t 1, t 2, t 3$ is shown in the fig. 31. All the four sensors has two sensing range $\mathrm{r} 1, \mathrm{r} 2$ where $\mathrm{r} 1<\mathrm{r} 2$. Here suppose a nodes sensing area is the circle where sensors are the centre with a radius equal to the sensing range. In fig. 32, a solid line denotes the range $r 1$ for each sensor and shaded line denotes the range $r 2$ for each sensor. Now the coverage relationships between sensors and targets are: $(\mathrm{s} 1, \mathrm{r} 1)=\mathrm{t} 3,(\mathrm{~s} 1, \mathrm{r} 2)=\mathrm{t} 1, \mathrm{t} 3$, $(\mathrm{s} 2, \mathrm{r} 1)=\mathrm{t} 2,(\mathrm{~s} 2, \mathrm{r} 2)=$

$\mathrm{t} 1, \mathrm{t} 2,(\mathrm{~s} 3, \mathrm{r} 1)=\mathrm{t} 2,(\mathrm{~s} 3, \mathrm{r} 2)=\mathrm{t} 2, \mathrm{t} 3,(\mathrm{~s} 4, \mathrm{r} 1)=\mathrm{t} 1, \mathrm{t} 3$ and

$(\mathrm{s} 4, \mathrm{r} 2)=\mathrm{t} 1, \mathrm{t} 2, \mathrm{t} 3$.

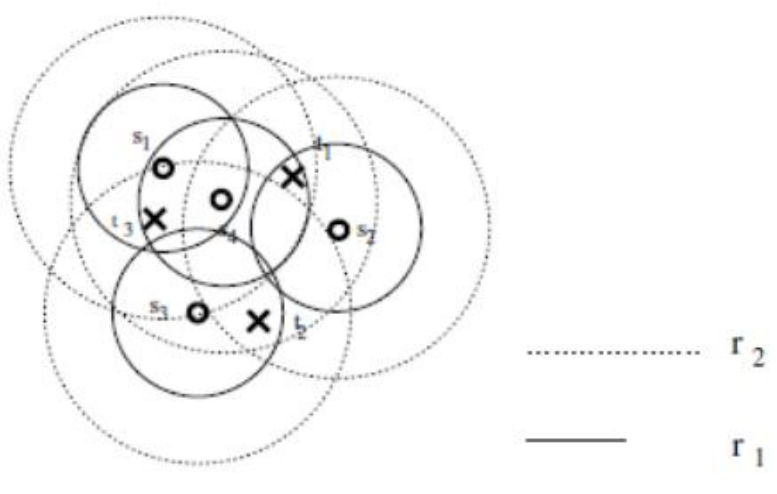

Fig. 32. A figure with three targets $\mathrm{T}=\mathrm{t} 1, \mathrm{t} 2, \mathrm{t} 3$ and four sensors $=\mathrm{s} 1, \mathrm{~s} 2, \mathrm{~s} 3, \mathrm{~s} 4$

A sensor can be part of more than one cover set and for a unit time of 1 each cover set is active. Now in the example, consider $\mathrm{E}=2$, e $1=0.5$ and $\mathrm{e} 2=1$. The AR-SC problem has one solution which utilizes the set covers explained in fig. 23. This solution has five various set covers C1, C2, C3, C4 and $\mathrm{C} 5$ where $\mathrm{C} 1=(\mathrm{s} 1, \mathrm{r} 1),(\mathrm{s} 2, \mathrm{r} 2), \mathrm{C} 2=(\mathrm{s} 1, \mathrm{r} 2),(\mathrm{s} 3, \mathrm{r} 1), \mathrm{C} 3=$ $(\mathrm{s} 2, \mathrm{r} 1),(\mathrm{s} 3, \mathrm{r} 2), \mathrm{C} 4=(\mathrm{s} 4, \mathrm{r} 2)$ and

$\mathrm{C} 5=(\mathrm{s} 1, \mathrm{r} 1),(\mathrm{s} 2, \mathrm{r} 1),(\mathrm{s} 3, \mathrm{r} 1)$ and this solution has maximum lifetime 6 with set covers order as C1, C2, C3, C4, C5 and $\mathrm{C} 4$. Here the set $\mathrm{C} 1$ has value $(\mathrm{s} 1, \mathrm{r} 1),(\mathrm{s} 2, \mathrm{r} 2)$ as in fig. 33 (a) the sensor $\mathrm{s} 1$ covers a sensing range $\mathrm{r} 1$ (solid line) and the sensor s2 covers the sensing range r2 (shaded line). Similarly, for the set C2 as shown in fig.33 (b), the sensor s1 covers the range $\mathrm{r} 2$ and sensor $\mathrm{s} 3$ covers the range $\mathrm{r} 1$. For set $\mathrm{C} 3$ as shown in fig. 33 (c), the sensor s2 covers the range $\mathrm{r} 1$ and sensor $\mathrm{s} 3$ covers the range $\mathrm{r} 2$. For the set $\mathrm{C} 4$ shown in fig. 33 (d), only the sensor s4 covers the range r2. For the set C5 shown in fig. 33 (e), the sensor s1, s2 and s3 covers the range $\mathrm{r} 1$. The remaining energy of each sensor is zero after the completion of this sequence.

A lifespan 4 for a sensing range same as $\mathrm{r} 2$ is derived if sensor nodes are not possessing adjustable sensing ranges. Set covers such as $(\mathrm{s} 1, \mathrm{r} 1),(\mathrm{s} 2, \mathrm{r} 2)$ and $(\mathrm{s} 4, \mathrm{r} 2)$ are the two distinct set covers in which sensors can be organized and each can be active twice. The residual energy values and the number of times a set cover is active are dependent on each other. Therefore, by applying adjustable sensing ranges the above example shows 50 .

\section{Connection between Coverage AND CONNECTIVITY}

The effective transmission of data is determined by connectivity. As described in [21] Coverage requires that in the coverage area all locations should be inside its sensing ranges where not less than one mobile node is there while connectivity requires only the location of 
one active node among one or more active nodes to be within the communication range such

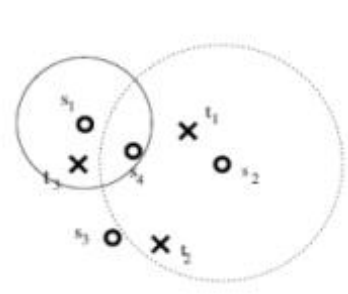

(a)

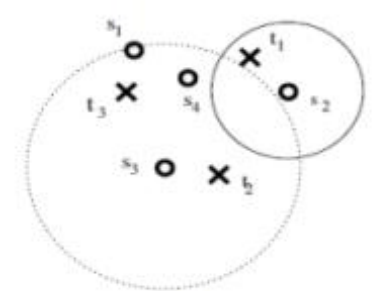

(c)

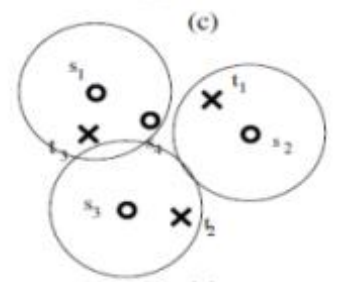

(e)

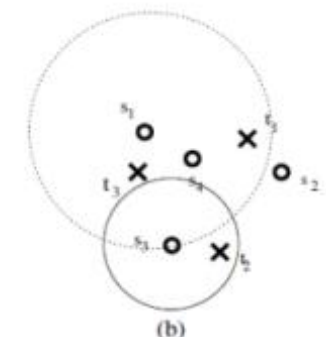

(b)

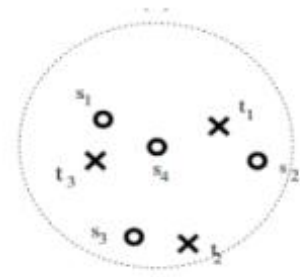

(d)
Fig. 33. Five set covers.

that communication backbone is formed by all mobile nodes. Selecting minimum number of mobile nodes and preserving coverage and maintaining connectivity is the aim of analysis on connection between coverage and connectivity.

The previous work of [21] gives the broad overview of the connection between coverage and connectivity. He has concluded in his work that if a set of nodes totally covers a convex, the communication graph including these nodes is joined when $\mathrm{Rc}>=2 \mathrm{Rs}$.

Suppose ' $A$ ' is the boundary of the convex sensor deployment region. A boundary sensor is a sensor whose sensing circle intersects with ' $A$ ' while interior sensors are all the sensors except boundary sensor in zone ' $A$ '. The boundary sensors are placed within Rs distance from ' $\mathrm{A}$ '.

Wang et al., [20] proved three theorems which are given as follows-

Lemma 1. If $\mathrm{Rc}>=2 \mathrm{Rs}$, it is possible to disconnect a boundary node from the remaining nodes in the communication graph by removing $\mathrm{k}$ sensors for a $\mathrm{K}$ covered area A which is convex.

Theorem 2. If Rc $>=2 \mathrm{Rs}$, then a set of nodes that K-cover a convex area A forms a K connected communication graph.
Theorem 3. If $\mathrm{Rc}>=2 \mathrm{Rs}$ then for a set of sensors that $\mathrm{K}$-cover an area A which is convex, the internal connectivity is $2 \mathrm{~K}$.

\section{3-DIMENSIONAL COVERAGE CONTROL ALGORITHM}

In 3-dimensional WSN, the optimal deployment is based on all the sensor nodes having identical transmission range and sensing range. In 3-dimensional space the position of sensor nodes are computed to ensure 100

A 3-dimensional shape which consists of finite number of polygonal faces is known as polyhedron. The faces of it meet in straight line segments called edges and the edges meet at points called vertices. Cube, prisms and pyramids are some examples of polyhedrons.

A polyhedron that can be used to fill a volume without any overlap or gap is a space-filling polyhedron. The sensing area of a node is spherical and spheres couldn't tessellate in $3 \mathrm{D}$, so a space-filling polyhedron which is the best approximation of a sphere is found out. In 3-dimensional WSN, the pictorial representation of a sensor node is a 0-1 Boolean pictorial representation. In 3-dimensional cuboids observed area, the sensor node set is $\mathrm{S}=\mathrm{s} 1, \mathrm{~s} 2, \ldots \mathrm{sN}$. Each sensor node $\mathrm{Si}$ where $\mathrm{i}=$ 1, . . . . . ., $\mathrm{N}$ with sensing range of $\mathrm{Rs}$ and a transmission range of Rt is located at coordinates (xi, yi, zi). So, Si the sensing area of each sensor node is a sphere centered at (xi, yi, zi) with radius

Rs and volume is $4 \mathrm{R} 2$. The target location that a sensor node focus is the grid. In space-filling polyhedron the centre grid set is $\mathrm{T}=\mathrm{t} 1, \mathrm{t} 2, \ldots \ldots, \mathrm{tN}$. Each grid ti is located at coordinate (xi, yi, zi). In fig.34 truncated octahedron has

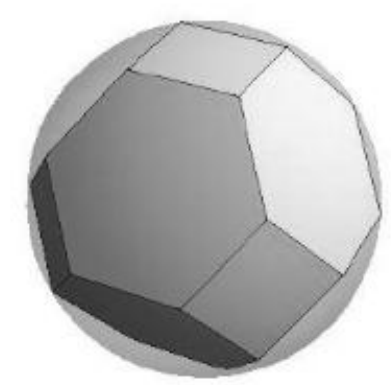

Fig. 34. Truncated octahedron and its circumsphere

14 faces including 6 square faces and 8 hexagonal faces. Suppose ' $a$ ' is the length of each edge. The distance between two opposite square faces is $2 \mathrm{a}$. The circumsphere 
radius of the $\operatorname{tr} \sqrt{ }$ uncated octahedron is,

$r=\frac{\sqrt{10 a}}{2}$

The volume of the truncated octahedron is $8 \sqrt{2 a^{3}}$ and the volumetric quotient is

$\frac{8 \sqrt{2 a^{3}}}{\frac{4 \pi r^{3}}{3}}=0.68329$

In 3-dimensional space if the space-filling polyhedron gives identical radius, then smaller the number of nodes for 3- dimensional complete coverage, higher will be the volumetric quotient of that polyhedron. As compared to other space-filling polyhedron, the volumetric quotient of truncated octahedron is much higher. For example, the volumetric quotient of rhombic dodecahedron is 0.477 ; the volumetric quotient of hexagonal prism is 0.477 , and cube has 0.36755 . So the truncated octahedron is discussed here.

Two truncated octahedrons tessellates with two conditions as shown in fig.35. The intersection face which is hexagonal face is one condition. Another is the square face.

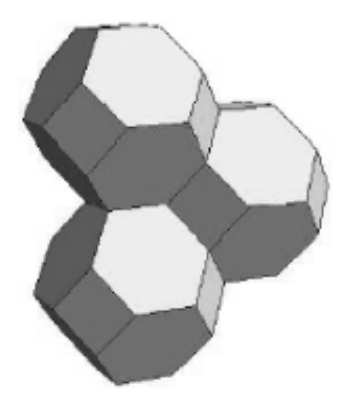

Fig. 35. Tessellation of the Truncated octahedrons

The detailed description of the conditions is given in [22].

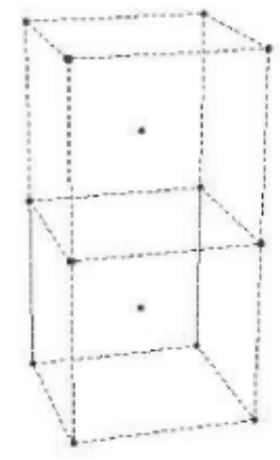

Fig. 36. Design of sensor node deployed in 3-dimensional space.

\section{CONCLUSION}

The two most fundamental issues in WSN are coverage and connectivity and they have a exceptional effect on Quality of service of WSNs. At first the essential knowledge of the idea of coverage is given in the paper. The paper introduces a wide review of coverage and connectivity and various meshes coverage efficiency is derived. In the paper various components like deployment of nodes, network characteristics and certain algorithms related to coverage and connectivity is covered. In 3-Dimensional space, various shapes volumetric quotient is calculated.

\section{FUTURE WORKS}

To enlarge the maximal lifespan planning of k-coverage and k-connectivity might be one of the future works. A further research can be done on improving the centralized server based algorithms and considering decentralized algorithms. Another further research can be done on combination of different kinds of sensors accompanied by different coverage strategy.

\section{REFERENCES}

[1] X Wang, S Zhang (2009) Research on efficient coverage problem of node in wireless sensor networks. Second International Symposium on Elec- tronic commerce and security 532-536. doi: 10.1109/ISECS.2009.248

[2] Bhagwan Sahay Meena, K. Hemachandran, Shaine Hussian Laskar,"Second International Conference on Information and Communication Technology for Competitive Strategies", Article No. 44 doi: 10.1145/2905055.2905250

[3] Bhagwan Sahay Meena, Sujoy Deb, K. Hemachandran, "Impact of hetrogenous IoT device's for indoor localization using RSSI", 4th International Conference on Research in Intelligent and Computing in Engineering , 2019

[4] S Ram , D Majunath, S Iyer, D Yogeshwaran (2007) On the path coverage properties of random sensor networks. IEEE Transaction on Mobile Computing 494-506.

[5] J Harada, S Shioda, H Saito (2008) Path coverage property of randomly deployed sensor networks with finite communication ranges. IEEE International conference on communication 2221-2227.

[6] S Kumar, T Lai, A Arora (2007) Barrier coverage with wireless sensors.

[7] 817-834. doi: 10.1007/s11276-006-9856-0.[7]B Liu, O Dousse, J Wang, A Saipulla (2008) Strong barrier coverage of wireless sensor networks. Proceedings of the 9th ACM International Symposium on mobile ad hoc networking and computing 411-420.

[8] A Chen, S Kumar, T.H. Lai (2007) Designing localized algorithms for barrier coverage. Proceedings of the 13th annual ACM International conference on mobile computing and networking 63-74.

[9] A Chen, T.H Lai, D Xuan (2008) Measuring and guaranteeing quality of barrier-coverage in wireless sensor networks. Proceedings of the 9th ACM international Symposium on mobile ad hoc networking and computing 421-430.

[10] F. Aurenhammer (1991) Voronoi diagrams. A survey of fundamental geometric data structure. ACM Computing Surveys 345-405.

[11] G Wang, G Cao, T La Porta (2004) Movement-assisted sensor deploy- ment. Twenty-third annual joint conference of the IEEE computer and Communication societies 2469-2479.

[12] D Wang, B Xie, D Agrawal (2008) Coverage and lifetime optimization of wireless sensor networks with gaussian distribution. IEEE Transac- tions on Mobile Computing 1444-1458.

[13] N Tamboli, M Younis (2009) Coverage-aware connectivity restoration in mobile sensor networks. IEEE International conference on Communication $1-5$. 
[14] P Sahoo, J.Z Tsai, H.L Ke (2010) Vector method based coverage hole recovery in wireless sensor networks. 2010 second International conference on Communication systems and networks (COMSNETS) 1-9.

[15] Y Zou, K Chakrabarty (2003) Sensor deployment and target localization based on virtual forces. Twenty-second annual joint conference of the IEEE computer and communication IEEE societies $1293-1303$.

[16] G Tan, S Jarvis and A.M Kermarrec (2009) Connectivity-guaranteed and obstacle-adaptive deployment schemes for mobile sensor networks. IEEE Transactions on Mobile Computing 836-848.

[17] D Tian, N.D Georganas (2002) A coverage-preserving node scheduling scheme for large wireless sensor networks. Proceedings of the 1st ACM International workshop on wireless sensor networks and applications 32-41.

[18] S Nath, P.B Gibbons (2007) Communicating via fireflies geographic routing on duty-cycled sensors. Proceedings of the 6th International conference on information processing in sensor networks ACM 440-449.

[19] M Cardei, M Thai, Y Li, W Wu (2005a) Energy-efficient target coverage in wireless sensor networks. Proceedings of 24th annual joint conference of the IEEE computer and Communication Societies 1976-1984.

[20] M Cardei, J Wu, M Lu, M Pervaiz (2005b) Maximum network lifetime in wireless sensor networks with adjustable sensing ranges. IEEE international conference on wireless and mobile computing networking and communications 438-445.

[21] X Wang, G Xing, Y Zhang, C Lu, R Pless (2003) Gill C. Integrated coverage and connectivity configuration in wireless sensor networks. Proceedings of the 1 st International conference on embedded networked sensor systems ACM 28-39.

[22] J Xiao, C Qi, L Shu (2012) Research of Coverage Control Algorithm in Three-Dimensional Wireless Sensor Network based on Energy Effi- ciency. 24th Chinese Control and Decision Conference (CCDC) 3363- 3368.

[23] J O'Rourke (1987) Art gallery theorems and algorithms. Oxford Uni- versity Press, Inc 